\title{
Association of Sarcopenia with Metabolic Syndrome in Korean Population Using 2009-2010 Korea National Health and Nutrition Examination Survey
}

\author{
Seong-Joon Park, MS, ${ }^{1}$ So-Yeon Ryu, MD, PhD, ${ }^{2}$ Jong Park, MD, PhD, ${ }^{2}$ and Seong-Woo Choi, MD, $\mathrm{PhD}^{2}$
}

\begin{abstract}
Background: Some studies have investigated the relationship between sarcopenia and metabolic syndrome, and they have focused mainly on older subjects. Therefore, we assessed the association between sarcopenia and metabolic syndrome in South Korean adults 20 years of age or older using data from the 2009-2010 Korean National Health and Nutrition Examination Survey (KNHANES).

Methods: This study involved 12,256 (5350 males and 6906 females) participants from the 2009-2010 KNHANES 20 years of age or older. Appendicular skeletal muscle mass (ASM) was measured by dual X-ray absorptiometry. Sarcopenia index (SI) was calculated as ASM/body mass index and sarcopenia was defined as an SI of $<0.789$ in males and $<0.521$ in females. Metabolic syndrome was defined by the presence of at least three of the following abnormalities: abdominal obesity, high blood pressure, high blood glucose level, high triglyceride level, and low high-density lipoprotein cholesterol level.

Results: After adjustment for covariates, the association between sarcopenia and metabolic syndrome was significant (odds ratio [OR] 2.06, 95\% confidence interval [CI] 1.74-2.45). In addition, when stratified by age groups, the significant associations between sarcopenia and metabolic syndrome remained in all age groups (20-39 years: OR $2.13,95 \%$ CI $1.08-4.19 ; 40-64$ years: OR $2.13,95 \%$ CI $1.68-2.71$; $\geq 65$ years: OR $1.98,95 \%$ CI 1.54-2.54).

Conclusion: The association between sarcopenia and metabolic syndrome was significant in South Korean adults. Moreover, the significant associations were present in every age group evaluated.
\end{abstract}

Keywords: sarcopenia, central obesity, dyslipidemia, hypertension, impaired fasting glucose

\section{Introduction}

$\mathrm{T}$ HE PREVALENCE OF METABOLIC SYNDROME is increasing globally. ${ }^{1}$ The prevalence was $\sim 34.7 \%$ in $2011-2012$ in the United States, ${ }^{2}$ and $\sim 23 \%$ of Japanese adults in their 30 s or older were diagnosed with metabolic syndrome in 2011. ${ }^{3}$ The prevalence of metabolic syndrome in Korea increased steadily from $27.5 \%$ in 2008 to $28.9 \%$ in $2013 .{ }^{4}$ As a result, the cost of medical treatment for metabolic syndrome in Korea increased from 3.7 trillion won in 2010 to 4.7 trillion won in 2014 , with an expected increase of $6.2 \%$ per year. ${ }^{5}$

Insulin resistance is the major cause of metabolic syndrome. ${ }^{6}$ As blood glucose levels increase in response to an increase in insulin resistance, insulin secretion further increases and results in hyperinsulinemia, and hyperinsulinemia restricts sodium excretion in the kidneys, resulting in hypertension. ${ }^{7}$ It also increases triglyceride (TG) and decreases high-density lipoprotein cholesterol (HDL-C) levels, resulting in dyslipidemia. ${ }^{8}$

Sarcopenia is defined as reductions in muscle mass and muscle strength due to changes in body composition. ${ }^{9}$ The prevalence of sarcopenia was estimated to be more than 50 million adults worldwide in 2000 and is expected to increase to more than 200 million by $2040 .^{10}$ The 2008-2011 Korea National Health and Nutrition Examination Survey (KNHANES) estimated the prevalence of sarcopenia in Korea to be $26.8 \% .^{11}$

The mechanism of sarcopenia has not yet been clarified, but it has been associated with various factors such as aging,

\footnotetext{
${ }^{1}$ Department of Public Health, Graduate School of Chosun University, Gwangju, Republic of Korea.

${ }^{2}$ Department of Preventive Medicine, Chosun University Medical School, Gwangju, Republic of Korea.

(C) Seong-Joon Park et al. 2019; Published by Mary Ann Liebert, Inc. This Open Access article is distributed under the terms of the Creative Commons Attribution Noncommercial License (http://creativecommons.org/licenses/by-nc/4.0/) which permits any noncommercial use, distribution, and reproduction in any medium, provided the original author(s) and the source are cited.
} 
malnutrition, lack of exercise, and reduced levels of hormones such as testosterone and cortisol. ${ }^{12}$ Furthermore, increased body fat resulting from decreased muscle mass seen in patients with sarcopenia is associated with cardiovascular and metabolic diseases. ${ }^{13}$ However, the relationship between sarcopenia and metabolic syndrome is still unclear. Several authors have reported an association between sarcopenia and metabolic syndrome, ${ }^{14}$ whereas others found no such association, ${ }^{15}$ and yet others reported that the association differs depending on sex. ${ }^{16}$ In addition, previous studies on the association between sarcopenia and metabolic syndrome have focused mainly on older subjects. ${ }^{14,17}$ However, the prevalence of metabolic syndrome is also increasing in younger populations. ${ }^{18}$ Therefore, we investigated the association between sarcopenia and metabolic syndrome in all adults in Korea using the 2009-2010 KNHANES.

\section{Materials and Methods}

\section{Study population}

This study used data from the 2009-2010 KNHANES. As previously published in detail, ${ }^{19}$ the KNHANES is a nationwide cross-sectional survey conducted annually by the Korea Centers for Disease Control and Prevention (KCDCP). The KNHANES uses a rolling sampling design involving a complex, stratified, multistage probabilitycluster survey of a representative sample of the noninstitutionalized Korean citizens residing in Korea. KNHANES consisted with three component surveys: the health interview, health examination, and nutrition survey. The health interview and nutrition survey questionnaires are administered by trained interviewers, and the health examinations are performed by trained medical staff. The KCDCP Ethics Committee approved the study protocol (2009-01CON-032C, 2010-02CON-21-C), and written informed consent was obtained from all subjects or their parents.

The number of participants in the 2009-2010 KNHANES was 19,491 (10,533 in 2009 and 8958 in 2010), among whom, 14,963 (7920 in 2009 and 7043 in 2010) people participated in both the health interview and the health examination, including whole body dual-energy X-ray absorptiometry (DXA) scans, and 13,201 were older than 20 years. In total, 12,256 participants (5350 males and 6906 females) were analyzed, after excluding 272 subjects with missing appendicular skeletal muscle mass (ASM) data and 673 who did not undergo blood testing.

\section{Data collection}

Trained investigators interviewed the subjects individually using a questionnaire. Monthly household income was divided into quartiles. Educational level was divided into $<6,7-9,10-12$, and $\geq 13$ years. Marriage status was classified as unmarried or married, and area of residence was classified as urban or rural. Current smoking was defined as smoking frequently or occasionally, and monthly drinking was defined as one or more drinks during the last month. Physical activity was defined as walking for $>30 \mathrm{~min}$ at a time at least six times per week. Strength training was defined as exercising the muscles more than once per week. Weight was measured to the nearest $0.1 \mathrm{~kg}$, while the subjects were dressed in light clothes, and height was measured to the nearest $0.1 \mathrm{~cm}$ in stocking feet. Waist circumference was measured to the nearest $0.1 \mathrm{~cm}$ at expiration through a horizontal plane around the abdomen midway between the lowest rib and iliac crest. Blood pressure was measured after the subject had rested for $5 \mathrm{~min}$ in a sitting position. Three readings each of systolic blood pressure (SBP) and diastolic blood pressure (DBP) were recorded, and the average values were used in the analyses. Number of co-morbidities was categorized as diagnosis of $0,1,2$, or $\geq 3$ of hypertension, diabetes, dyslipidemia, stroke, myocardial infarction, angina pectoris, cancer, cirrhosis, and kidney failure. The blood samples were collected by a trained nurse and transported daily to the central laboratory of NEODIN Medical Institute (Seoul, Korea). The total cholesterol, TG, and HDL-C levels were determined using the Hitachi Automatic Analyzer 7600 (Hitachi Ltd., Tokyo, Japan) according to standard procedures.

\section{Sarcopenia}

ASM was measured by dual X-ray absorptiometry (QDR 4500A; Hologic, Inc., Bedford, MA). The sarcopenia index (SI) was calculated as ASM $(\mathrm{kg}) /$ body mass index (BMI, $\mathrm{kg} / \mathrm{m}^{2}$ ), and sarcopenia was defined as an SI of $<0.789$ in males and $<0.521$ in females based on the criteria of the Sarcopenia Project. ${ }^{20}$

\section{Metabolic syndrome}

A diagnosis of metabolic syndrome was defined as the presence of three or more of the following five components: high blood pressure, high blood glucose level, high TG level, and lower HDL-C level using the diagnostic criteria of the National Cholesterol Education Program Adult Treatment panel III (NCEP-ATP III) based on common clinical measures,$^{21}$ and the abdominal obesity using the criteria of the Korean Society for the Study of Obesity.22 Among the metabolic syndrome components, abdominal obesity was defined as a waist circumference $\geq 90 \mathrm{~cm}$ for males or $\geq 80 \mathrm{~cm}$ for females, high blood pressure as SBP $\geq 130 \mathrm{mmHg}$ or DBP $\geq 85 \mathrm{mmHg}$ or the use of antihypertensive medication, high blood glucose as a fasting blood glucose (FBG) level $\geq 100 \mathrm{mg} / \mathrm{dL}$ or the use of diabetic medication, a high TG level as $\geq 150 \mathrm{mg} / \mathrm{dL}$ or the use of dyslipidemia medication, and a low HDL-C level as $<40 \mathrm{mg} / \mathrm{dL}$ for males or $<50 \mathrm{mg} / \mathrm{dL}$ for females, or the use of dyslipidemia medication.

\section{Statistical analysis}

Data were analyzed using SPSS (version 23.0; IBM, Armonk, NY). The survey responses were weighted by reference to the multistage, complex, probability sampling design. Data were expressed as absolute number and estimated percentages (with standard errors) or as mean \pm standard deviation (SD). The survey responses were weighted by reference to the multistage, complex probability sampling design. The $\chi^{2}$ test or Student's $t$-test was used to evaluate the differences in demographic and clinical characteristics according to sarcopenia. A multivariate logistic regression analysis was performed to investigate the association of sarcopenia with metabolic syndrome. Adjustment for sex, age, monthly household income, educational level, marital status, area of residence, current smoking, monthly drinking, physical activities and strength training, and number of comorbidities were performed. A value of $P<0.05$ was considered indicative of statistical significance. 


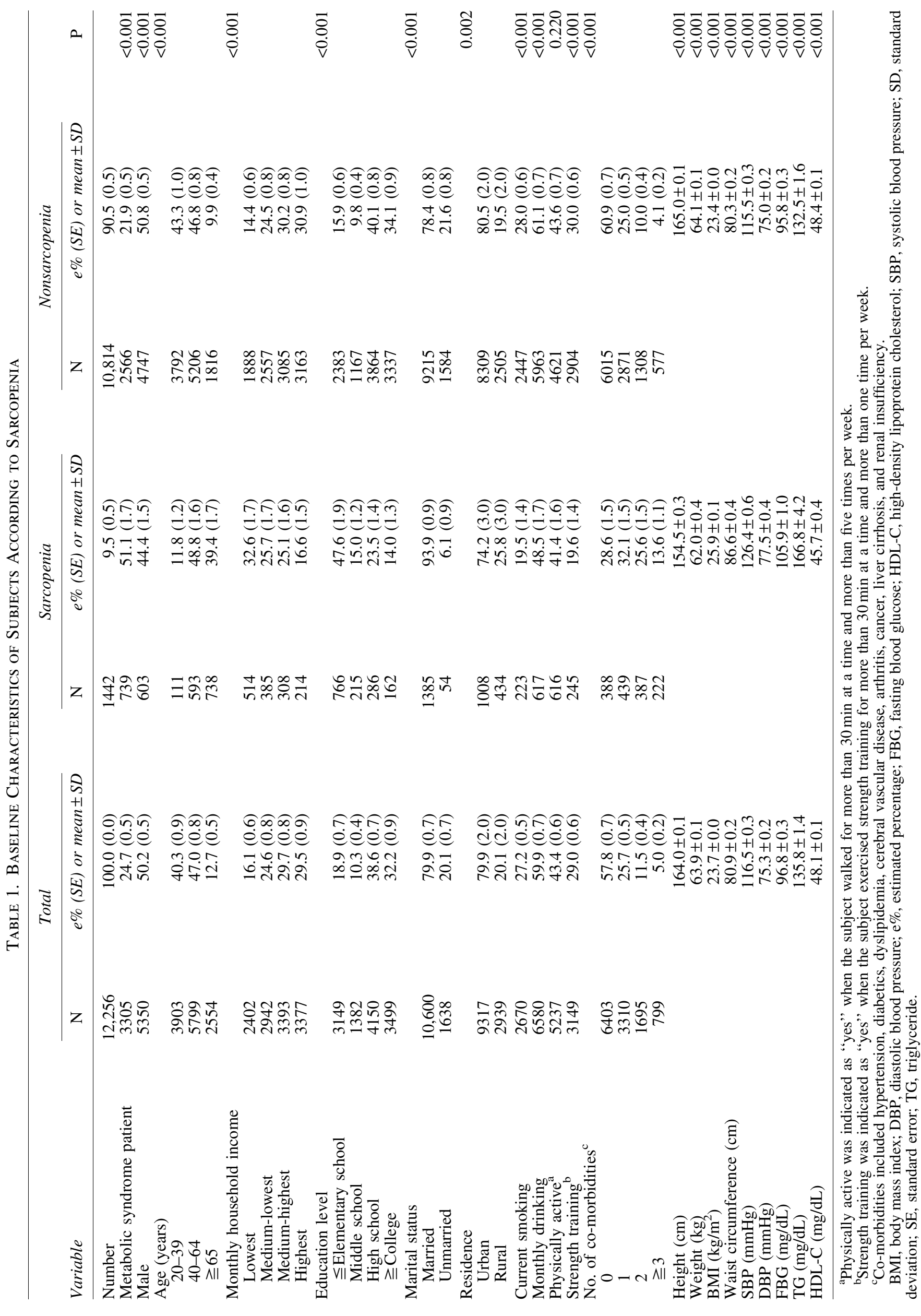




\section{Results}

\section{Baseline characteristics of subjects according to sarcopenia}

The baseline characteristics of subjects according to sarcopenia are shown in Table 1. The prevalence of sarcopenia and metabolic syndrome was $9.5 \%$ and $24.7 \%$. Among the subjects, $27.2 \%$ currently smoked, $59.9 \%$ had consumed alcohol in the past month, and $43.4 \%$ were physically active. The subjects with sarcopenia were older, had a lower monthly household income, had a lower educational level, were predominantly married, resided mainly in a rural area, smoked and drank less, and performed less strength training than those with nonsarcopenia (all $P<0.001$, except $P=0.002$ for residing in a rural area). The mean BMI, waist circumference, and levels of SBP, DBP, FBG, and TG were higher, whereas the HDL-C level was lower, in the subjects with sarcopenia than in those with nonsarcopenia (all $P<0.001$ ).

\section{Prevalence of metabolic syndrome according to sarcopenia}

The prevalence of metabolic syndrome according to sarcopenia is shown in Table 2. The five components of metabolic syndrome (abdominal obesity, high blood pressure, high blood glucose level, high TG level, and low HDL-C level) were all higher in subjects with sarcopenia than in those with nonsarcopenia (all $P<0.001$ ). In addition, metabolic syndrome prevalence was higher in the subjects with sarcopenia than in those with nonsarcopenia $(P<0.001)$.

\section{Odds ratios for sarcopenia according to metabolic syndrome}

The odds ratios (ORs) for sarcopenia according to metabolic syndrome are shown in Table 3. The crude ORs for sarcopenia were statistically significant (OR 3.73, 95\% confidence interval [CI $3.21-4.33$ ). After adjusting for sex, age, monthly household income, education level, marital status, residence, current smoking, monthly drinking, physical activities, strength training, and number of co-morbidities, the OR showed that the significant association between metabolic syndrome and sarcopenia remained (OR 2.06, 95\% CI 1.74-2.45).

Table 2. Prevalence of Metabolic Syndrome ACCORDING TO SARCOPENIA

\begin{tabular}{lccc}
\hline Variable & Sarcopenia & Nonsarcopenia & $\mathrm{P}$ \\
\hline Abdominal obesity $^{\mathrm{a}}$ & $49.2(2.1)$ & $22.4(0.6)$ & $<0.001$ \\
Higher blood $^{\text {pressure }}$ & $54.6(1.7)$ & $29.3(0.7)$ & $<0.001$ \\
Higher blood glucose $^{\mathrm{c}}$ & $45.9(1.5)$ & $24.0(0.6)$ & $<0.001$ \\
Higher triglyceride $^{\mathrm{d}}$ & $43.6(1.6)$ & $27.3(0.5)$ & $<0.001$ \\
Lower HDL-C $^{\mathrm{e}}$ & $54.3(1.7)$ & $41.1(0.6)$ & $<0.001$ \\
Metabolic syndrome & $51.3(1.7)$ & $21.9(0.5)$ & $<0.001$ \\
\hline
\end{tabular}

All values are presented as estimated percentage (SE).

${ }^{a}$ Abdomal obesity is defined as waist circumference $\geq 90 \mathrm{~cm}$ (male) or $\geq 80 \mathrm{~cm}$ (female).

${ }^{\mathrm{b}}$ Higher blood pressure is defined as SBP $\geq 130 \mathrm{mmHg}$ or DBP $\geq 85 \mathrm{mmHg}$.

${ }^{c}$ Higher blood glucose is defined as $F B G \geq 100 \mathrm{mg} / \mathrm{dL}$.

${ }^{\mathrm{d}}$ Higher triglyceride is defined as $\mathrm{TG} \geq 150 \mathrm{mg} / \mathrm{dL}$.

${ }^{\mathrm{e}}$ Lower HDL-C is defined as HDL-C $<40 \mathrm{mg} / \mathrm{dL}$ (male) or $<50 \mathrm{mg}$ / dL (female).
TABle 3. Odds Ratios for SARCOPEnia According to Metabolic Syndrome

\begin{tabular}{|c|c|c|}
\hline & Nonadjusted & Adjusted $^{\mathrm{a}}$ \\
\hline Variables & OR $(95 \% C I)$ & OR $(95 \% C I)$ \\
\hline $\begin{array}{l}\text { Nonmetabolic } \\
\text { syndrome }\end{array}$ & Reference & Reference \\
\hline Metabolic syndrome & $3.73(3.21-4.33)$ & $2.06(1.74-2.45)$ \\
\hline
\end{tabular}

${ }^{a}$ Adjusted by sex, age, monthly household income, education level, marital status, residence, current smoking, monthly drinking, physical activities, strength training, and number of co-morbidities.

CI, confidence interval; OR, odds ratio.

\section{ORs for sarcopenia according to metabolic syndrome stratified by age group}

The ORs for sarcopenia according to metabolic syndrome stratified by age group are shown in Table 4 . After adjusting for same covariates, the association between metabolic syndrome and sarcopenia was significant in subjects 20-39 years (OR 2.13, 95\% CI 1.08-4.19), 40-64 years (OR 2.13, $95 \%$ CI 1.68-2.71), and $\geq 65$ years (OR 1.98, 95\% CI $1.54-$ 2.54) of age.

\section{Discussion}

This study investigated the relationship between sarcopenia and metabolic syndrome in Korean adults using data from the 2009-2010 KNHANES. After adjustment for covariates, we found that sarcopenia was significantly associated with metabolic syndrome. In addition, after stratifying the study population by age, this significant association remained in all age groups.

There is no consensus on clinical diagnostic standards for sarcopenia, and previous researchers have used different diagnostic criteria: ASM/height ${ }^{2},{ }^{23}$ ASM/weight, ${ }^{24}$ and ASM/BMI. ${ }^{20}$ Baumgartner et al. $^{23}$ diagnosed sarcopenia as an $\mathrm{ASM} /$ height $^{2}>2$ SD below the mean in a young reference population and they reported that sarcopenia prevalence of $14 \%$ and $>50 \%$ in those $65-69$ and $\geq 80$ years of age, respectively, in New Mexico. Janssen et al. ${ }^{24}$ defined sarcopenia using ASM/weight and the sarcopenia prevalence was $7 \%$ and $11 \%$ in male and female $\geq 80$ years of age. A problem associated with the various diagnostic methods in prior studies is the selection of a young healthy reference population. In addition, the ability to compare results is limited since the reference populations differ among studies. Therefore, to clarify the diagnostic criteria for sarcopenia, the Foundation for the National Institutes of Health (FNIH) Sarcopenia Project used data from nine cohorts and defined sarcopenia as an ASM/BMI of $<0.789$ for males and $<0.521$ for females, without comparison to a young reference population. ${ }^{20}$

The prevalence of sarcopenia in South Koreans differed from study to study due to various diagnostic criteria. In this study using the FNIH definition, we found that the prevalence of sarcopenia was $9.5 \%$ (male $8.4 \%$ and female $10.7 \%$ ) in $\geq 20$ years of age and $14.1 \%$ (male $12.7 \%$ and female $15.4 \%$ ) in $\geq 40$ years of age. In a study using 2008 2009 KNHANES data, sarcopenia was defined according to two methods: ASM/height ${ }^{2}$ and ASM/weight that was greater than 2 SD below the mean in healthy individuals 20 
Table 4. Odds Ratios for Sarcopenia According to Metabolic Syndrome Stratified by Age Group

\begin{tabular}{|c|c|c|c|}
\hline \multirow[b]{2}{*}{ Age (years) } & \multirow[b]{2}{*}{ Metabolic syndrome } & \multirow{2}{*}{$\begin{array}{l}\text { Nonadjusted } \\
\text { OR }(95 \% \text { CI })\end{array}$} & \multirow{2}{*}{$\begin{array}{c}\text { Adjusted }^{\mathrm{a}} \\
\text { OR }(95 \% C I)\end{array}$} \\
\hline & & & \\
\hline \multirow[t]{2}{*}{ 20-39 } & Nonmetabolic syndrome & Reference & Reference \\
\hline & Metabolic syndrome & $3.42(2.08-5.63)$ & $2.13(1.08-4.19)$ \\
\hline \multirow[t]{2}{*}{$40-64$} & Nonmetabolic syndrome & Reference & Reference \\
\hline & Metabolic syndrome & $2.53(2.06-3.11)$ & $2.13(1.68-2.71)$ \\
\hline \multirow[t]{2}{*}{$\geq 65$} & Nonmetabolic syndrome & Reference & Reference \\
\hline & Metabolic syndrome & $2.07(1.68-2.55)$ & $1.98(1.54-2.54)$ \\
\hline
\end{tabular}

${ }^{a}$ Adjusted by sex, monthly household income, education level, marital status, residence, current smoking, monthly drinking, physical activities, strength training, and number of co-morbidities.

39 years of age. The prevalence of sarcopenia in males and females $>40$ years of age was $12.4 \%$ and $0.1 \%$ according to $\mathrm{ASM} /$ height $^{2}$ and $9.7 \%$ and $11.8 \%$ according to ASM/weight, respectively. ${ }^{25}$ In another study based on 2008-2011 KNHANES data, sarcopenia was defined according to ASM/weight, and the prevalence of sarcopenia in subjects $>20$ years of age was $26.8 \%,{ }^{11}$ which was significantly higher than our results $(9.5 \%)$. The reason for the higher prevalence of sarcopenia is that the authors used an ASM/weight $>1$ SD below the mean in a young reference population instead of an ASM/weight $>2 \mathrm{SD}$ as the diagnostic criteria for sarcopenia.

In this study, after adjustment for other covariates, the association between sarcopenia and metabolic syndrome was significant. In a meta-analysis of middle-aged and older nonobese adults, ${ }^{26}$ sarcopenia was significantly associated with metabolic syndrome (OR 2.01, 95\% CI 1.63-2.47), which is consistent with our results. In a previous study involving 1971 elderly Japanese subjects $\geq 65$ years of age, ${ }^{16}$ metabolic syndrome was significantly associated with sarcopenia in males (OR 2.08, 95\% CI 1.22-3.54), but not in females. In Korea, 4183 postmenopausal females were analyzed using the 2008-2011 KNHANES data, ${ }^{14}$ and a statistically significant relationship was identified between sarcopenia and metabolic syndrome (OR 1.97, 95\% CI 1.51-2.56).

In this study, after stratifying the study population by age, the association between sarcopenia and metabolic syndrome was significant in all age groups evaluated (20-39, 40-64, and $\geq 65$ years). In a previous study of 5300 adults 19-39 years of age, which used 2008-2010 KNHANES data, low muscle mass was defined according to ASM/weight and was significantly associated with metabolic syndrome in young adults, which is consistent with our results. ${ }^{27}$ Most previous studies have evaluated the relationship between sarcopenia and metabolic syndrome in the elderly because muscle mass and muscle strength are assumed to decrease the most with age. However, it has been reported that the percentage of total lean body mass begins to decrease starting in the early 30 s, and that sarcopenia can occur in younger populations. ${ }^{28}$ However, in the case of sarcopenia, the pattern varies according to age. Sarcopenia in the elderly is accompanied by selective atrophy of type II muscle fibers, whereas sarcopenia in young adults is accompanied by an overall decrease in muscle mass. ${ }^{29}$

Metabolic syndrome consists of five components: abdominal obesity, high blood pressure, high blood glucose level, high TG level, and low HDL-C level. Insulin resistance and inflammation are considered the central mechanisms responsible for metabolic syndrome. ${ }^{30}$ Several mechanisms may underlie the association between sarcopenia and metabolic syndrome. First, skeletal muscle is the most important organ for systemic glucose homeostasis ${ }^{31}$ and is responsible for $\sim 80 \%$ of normal glucose absorption and metabolism by insulin stimulation under normal conditions. ${ }^{32}$ Second, a decrease in muscle mass leads to an increase in fat mass by reducing the basal metabolic rate. ${ }^{33}$ Increased levels of fat increase the secretion of inflammatory cytokines such as tumor necrosis factor-alpha and interleukin (IL)- $6^{34}$ and increase insulin resistance, which can eventually lead to metabolic syndrome. ${ }^{31,35}$ Third, skeletal muscle cells express and secrete many myokines, including IL-6, IL-8, IL-15, fibroblast growth factor 21 , irisin, myonectin, and myostatin. ${ }^{36}$ Most myokines are controlled primarily by exercise and muscle exertion. They offset the deleterious effects of inflammatory cytokines and have beneficial effects on glucose and lipid metabolism as well as inflammation. ${ }^{37}$

The limitations of this study are as follows. First, it was difficult to clarify causality because of the cross-sectional design. Second, a new guideline of the European Working Group on Sarcopenia in Older People in 2018 recommended measuring muscle strength as well as muscle mass to diagnose sarcopenia. ${ }^{10}$ However, the 2009-2010 KNHANES did not measure muscle strength. Nevertheless, this study is meaningful in that, it investigated the relationship between sarcopenia and metabolic syndrome in all adults older than 20 years using representative data from Korea.

In conclusion, after adjusting for covariates, the association between sarcopenia and metabolic syndrome was significant in South Korean adults. Moreover, after stratifying by age groups, the significant associations between sarcopenia and metabolic syndrome remained in all age groups.

\section{Author Disclosure Statement}

No conflicting financial interests exist.

\section{Funding Information}

This study was supported by research fund from Chosun University, 2018.

\section{References}

1. Grundy SM. Metabolic syndrome pandemic. Arterioscler Thromb Vasc Biol 2008;28:629-636.

2. Aguilar M, Bhuket T, Torres S, et al. Prevalence of the metabolic syndrome in the United States, 2003-2012. JAMA 2015;313:1973-1974.

3. Kim J, Tanabe K, Yokoyama N, et al. Objectively measured light-intensity lifestyle activity and sedentary time 
are independently associated with metabolic syndrome: A cross-sectional study of Japanese adults. Int J Behav Nutr Phys Act 2013;10:30.

4. Tran BT, Jeong BY, Oh JK. The prevalence trend of metabolic syndrome and its components and risk factors in Korean adults: Results from the Korean National Health and Nutrition Examination Survey 2008-2013. BMC Public Health 2017;17:71.

5. Statistics Korea, 2015 Korean social trends. Daejeon: Statistical Research Institute; 2015.

6. Gami AS, Witt BJ, Howard DE, et al. Metabolic syndrome and risk of incident cardiovascular events and death: A systematic review and meta-analysis of longitudinal studies. J Am Coll Cardiol 2007;49:403-414.

7. Reaven GM, Laws A. Insulin resistance, compensatory hyperinsulinaemia, and coronary heart disease. Diabetologia 1994;37:948-952.

8. Choi CS. Pathogenesis of insulin resistance. Korean J Med 2009;77:171-177.

9. Rosenberg IH. Sarcopenia: Origins and clinical relevance. J Nutr 1997;127:990S-991S.

10. Cruz-Jentoft AJ, Baeyens JP, Bauer JM, et al. Sarcopenia: European consensus on definition and diagnosis: Report of the European Working Group on Sarcopenia in Older People. Age Ageing 2010;39:412-423.

11. Bae EJ, Kim YH. Factors affecting Sarcopenia in Korean adults by age groups. Osong Public Health Res Perspect 2017;8:169-178.

12. Wang C, Bai L. Sarcopenia in the elderly: Basic and clinical issues. Geriatr Gerontol Int 2012;12:388-396.

13. Kim KM, Lim S, Choi SH, et al. Cardiometabolic implication of sarcopenia: The Korea National Health and Nutrition Examination Study (KNHANES) 2008-2010. IJC Metab Endocr 2014;4:63-69.

14. Kang SY, Lim GE, Kim YK, et al. Association between sarcopenic obesity and metabolic syndrome in postmenopausal women: A cross-sectional study based on the Korean National Health and Nutritional Examination Surveys from 2008 to 2011. J Bone Metab 2017;24:9-14.

15. Scott D, Park MS, Kim TN, et al. Associations of low muscle mass and the metabolic syndrome in Caucasian and Asian middle-aged and older adults. J Nutr Health Aging 2016;20:248-255.

16. Ishii S, Tanaka T, Akishita M, et al. Metabolic syndrome, sarcopenia and role of sex and age: Cross-sectional analysis of Kashiwa cohort study. PLoS One 2014;9:e112718.

17. Lu CW, Yang KC, Chang HH, et al. Sarcopenic obesity is closely associated with metabolic syndrome. Obes Res Clin Pract 2013;7:e301-e307.

18. Lim S, Shin H, Song JH, et al. Increasing prevalence of metabolic syndrome in Korea: The Korean National Health and Nutrition Examination Survey for 1998-2007. Diabetes Care 2011;34:1323-1328.

19. Kweon S, Kim Y, Jang MJ, et al. Data resource profile: The Korea National Health and Nutrition Examination Survey (KNHANES). Int J Epidemiol 2014;43:69-77.

20. Studenski SA, Peters KW, Alley DE, et al. The FNIH sarcopenia project: Rationale, study description, conference recommendations, and final estimates. J Gerontol A Biol Sci Med Sci 2014;69:547-558.

21. Grundy SM, Cleeman JI, Daniels SR, et al. Diagnosis and management of the metabolic syndrome: An American Heart Association/National Heart, Lung, and Blood Institute Scientific Statement. Circulation 2005;112:2735-2752.

22. Oh SW. Obesity and metabolic syndrome in Korea. Diabetes Metab J 2011;35:561-566.
23. Baumgartner RN, Koehler KM, Gallagher D, et al. Epidemiology of sarcopenia among the elderly in New Mexico. Am J Epidemiol 1998;147:755-763..

24. Janssen I, Heymsfield SB, Ross R. Low relative skeletal muscle mass (sarcopenia) in older persons is associated with functional impairment and physical disability. J Am Geriatr Soc 2002;50:889-896.

25. Kim YS, Lee Y, Chung YS, et al. Prevalence of sarcopenia and sarcopenic obesity in the Korean population based on the Fourth Korean National Health and Nutritional Examination Surveys. J Gerontol A Biol Sci Med Sci 2012;67: 1107-1113.

26. Zhang H, Lin S, Gao T, et al. Association between sarcopenia and metabolic syndrome in middle-aged and older non-obese adults: A systematic review and meta-analysis. Nutrients 2018;10:364.

27. Kim BC, Kim MK, Han K, et al. Low muscle mass is associated with metabolic syndrome only in nonobese young adults: The Korea National Health and Nutrition Examination Survey 2008-2010. Nutr Res 2015;35:10701078.

28. Gallagher D, Visser M, De Meersman RE, et al. Appendicular skeletal muscle mass: Effects of age, gender, and ethnicity. J Appl Physiol 1997;83:229-239.

29. Moon SS. Low skeletal muscle mass is associated with insulin resistance, diabetes, and metabolic syndrome in the Korean population: The Korea National Health and Nutrition Examination Survey (KNHANES) 2009-2010. Endocr J 2014;61:61-70.

30. Romeo Giulio R, Lee J, Shoelson Steven E. Metabolic syndrome, insulin resistance, and roles of inflammationMechanisms and therapeutic targets. Arterioscler Thromb Vasc Biol 2012;32:1771-1776.

31. Petersen KF, Dufour S, Savage DB, et al. The role of skeletal muscle insulin resistance in the pathogenesis of the metabolic syndrome. Proc Natl Acad Sci U S A 2007;104: 12587-12594.

32. Petersen KF, Shulman GI. Pathogenesis of skeletal muscle insulin resistance in type 2 diabetes mellitus. Am J Cardiol 2002;90:11G-18G.

33. Roubenoff R. Sarcopenic obesity: Does muscle loss cause fat gain? Lessons from rheumatoid arthritis and osteoarthritis. Ann N Y Acad Sci 2000;904:553-557.

34. Schrager MA, Metter EJ, Simonsick E, et al. Sarcopenic obesity and inflammation in the InCHIANTI study. J Appl Physiol 2007;102:919-925.

35. Reaven GM. Banting lecture 1988. Role of insulin resistance in human disease. Diabetes 1988;37:1595-1607.

36. Wu H, Ballantyne CM. Skeletal muscle inflammation and insulin resistance in obesity. J Clin Invest 2017;127:43-54.

37. Eckardt K, Görgens SW, Raschke S, et al. Myokines in insulin resistance and type 2 diabetes. Diabetologia 2014; 57:1087-1099.

Address correspondence to:

Seong-Woo Choi, $M D, P h D$

Department of Preventive Medicine Chosun University Medical School 309, Pilmun-daero

Dong-gu

Gwangju 61452

Republic of Korea

E-mail: jcsw74@hanmail.net 